\title{
Research on Battery Box Lightweight Based on Material Replacement
}

\author{
Qiu-Sheng Chen ${ }^{1, a, *}$, Han Zhao ${ }^{1, b}$, Ling-Xue Kong, ${ }^{2, c}$, Kang-Wei Chen ${ }^{3, d}$ \\ ${ }^{1}$ School of Mechanical Engineering,Hefei University of Technology,Hefei 230009, \\ China; \\ ${ }^{2}$ Institute for Frontier Materials,Deakin University, Geelong Victoria 3220, Australia; \\ ${ }^{3}$ New EnergyVehicle R\&D Institure, Anhui jianghuai automobile group co., \\ LTD,Hefei 230601, China; \\ a774792703@qq.com, bhanzhaoff@qq.com, \\ clingxue.kong@deakin.edu.au, ${ }^{\mathrm{d} z j z . x n y . j s z x @ j a c . c o m . c n ~}$
}

\begin{abstract}
Battery box lightweighting and stiffness are two important objectives of battery box design and improvement. In order to reduce a battery box's weight without impairment of stiffness, a method of battery box parts' material replacement from mild steel sheet to Aluminium alloy steel sheet of changed depth is brought forward,the selection of the appropriate material by using the material index is studied.The thickness change equation is drawn from structural mechanical analysis and verified by finite element simulation of body parts. Before and after the material replacement,the displacement nephogram and stress nephogram is obtained through simulation analysis of the battery box and there is no obvious difference between them show that the method presented in this paper is valid.
\end{abstract}

Keywords:battery box, Aluminium alloy,lightweighting, material replacement, stiffness

\section{Introduction}

With the rapid development of the global economy and society, energy depletion, environmental pollution, the study of new energy vehicles has become the focus of automotive industry[1].It is the international consensus of realizing automobile power electrification and developing electric vehicles. Battery box assembly occupies an important position in the structure of new energy vehicles[2].At present, the single battery energy density is relatively small, in order to achieve sufficient mileage and power performance, the quantities of battery are large, cause the battery box assembly is overweight, which affects the speed and mileage of the electric vehicle performance. Furthermore, the battery box assembly accounts for 25\%-30\% of the total weight of the car, consumes a large amount of battery energy, so reducing the weight of the battery box has become the urgent task of the development of new energy vehicles.

DONG Xianglong[3] improved and optimized the local structures of the battery box, thus improved the stress concentration on the local structures, the structure strength of 
the battery box has been improved, the weight is reduced. Hartmann[4] use finite element software optimizing the battery box's structure, the natural frequency of the battery box is improved, the battery box wall thickness is reduced, the weight of the battery box is reduced by $20 \%$ on the basis of the original structures. Although these scholars optimized the structure of the electric vehicle, and reduced the weight of the metal battery box successfully, but still can not change the fact that the battery box is too heavy.The battery box lightweighting can be achieved by structure modification or material replacement. The battery box structure modification requires the changes of forming, welding and assembling systems which is costly, while material replacement needs fewer such changes. Furthermore,material replacement is generally more effective in automobile lightweighting than structure modification. Therefore,only the battery box lightweighting by material replacement is studied in this paper.

In order to reduce the battery box weight,aluminum alloy,high strength steel, composite material, and so on, are widely used as lightweighting materials to replace the traditional material of mild steel[5,6].In these materials, aluminum alloy is a very good representative. The battery box weight can be reduced by use of Aluminium alloy sheet to replace the mild steel sheet of box parts. In order to retain the stiffness of the battery box, each box part which has the material changed should retain its stiffness. This paper will study the aluminum alloy sheet depth determination method with a battery box as an example,the selection of suitable materials by material index is studied,displacement nephogram and stress nephogram are obtained through the simulation analysis and the comparison is carried out.

\section{Material selection}

From the mechanical point of view, structural design is an important step in product design, and another important step is the process of material selection.Its primary objective is to determine the required properties of the material to be used, and then select the actual material to filter out the most suitable material from a variety of available materials.It is very important to choose the right material in the process of product formation.Since the selection of material is interwoven with all other aspects of the product formation process, it usually does not occur at the beginning or the end of the process, but in all stages.

It is very helpful to pre-select in the design stage, which can be refined in the following process.As the name of the lightweight structure is shown, the weight should be as low as possible, and it must also be able to meet certain set requirements for rigidity and mechanical strength. Therefore, in the selection of materials, emphasis should be placed on the densityp.If the parts' stiffness is concerned more, it should focus on the elastic modulus E, but they always should be considered at the same time . 


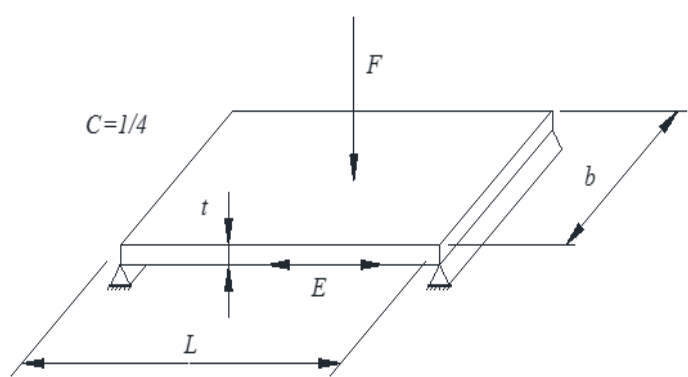

Fig.1 Deflection of a plate

The battery box is used to bear the weight of the battery pack, which is welded by a piece of plate.As shown in Figure 1,A flat plate, which main bearing bending stress, it should have a set of minimum stiffness, the length $L$ and width $b$ of the plate are known, in this case, the lightweight structure of the objective equation is:

$$
m=A t \rho=L b t \rho
$$

The deformation of the plate under the load $F_{\max }$ must not exceed the maximum deflection $\delta_{c}$, at this point, the stiffness requirements can be expressed by the following formula:

$$
C \frac{F_{\max } L^{3}}{E t^{3} b} \leq \delta_{C}
$$

where, $\mathrm{C}$ is a constant that is related to the form of a specific load, and joint formula (1) and (2),we can obtain the following formula:

$$
m \geq \sqrt[3]{\frac{C F_{\max } L^{6} b^{2}}{\delta_{C}}} \frac{\rho}{\sqrt[3]{E}}
$$

As a result, it can be seen from the formula (3) that in the case of $C, F_{\max }, \mathrm{L}, \mathrm{b}$ are not changed, in order to make the quality of the plate is minimum,we should make the $\frac{\rho}{\sqrt[3]{E}}$ stress should use the material index $M=\frac{\sqrt[3]{E}}{\rho}$ as high as possible.Material index $\mathrm{M}$ is a measure of the combination of material properties, indicating the suitability of the material for a particular application.The following is a comparison of the material index of steel, aluminum, magnesium and titanium alloys:

Table 1 Comparison of material index of various materials

\begin{tabular}{|c|c|c|c|}
\hline Material & Aluminium & titanium alloy & steel \\
\hline Elastic model E/GPa & 72 & 115 & 210 \\
\hline density $\rho /\left(\mathrm{mg} \cdot \mathrm{m}^{-3}\right)$ & 2.74 & 4.5 & 7.9 \\
\hline $\boldsymbol{M}=\frac{\sqrt[3]{\boldsymbol{E}}}{\boldsymbol{P}}$ & 1.54 & 1.08 & 0.75 \\
\hline
\end{tabular}

As can be seen from the above table, the use of aluminum alloy as a plate material has an absolute advantage in the light weight than the steel. 


\section{Battery box part thickness analysis}

\subsection{Thickness selection of the same stiffness}

If the elastic modulus of a material is less than that of steel, it is necessary to take into account that the structure of the material will produce greater deformation in the design of the bearing, it is important to pay attention to this point when using aluminum alloy to replace the steel. Battery box is mainly used to withstand the weight of the battery, the bearing structure need to meet the deformation conditions. the elastic modulus of aluminum is only $1 / 3$ of the elastic modulus of steel due to the deformation is 3 times than that of steel. A single load analysis in the center is used to investigate the deflection of a plate ${ }^{[5]}$.

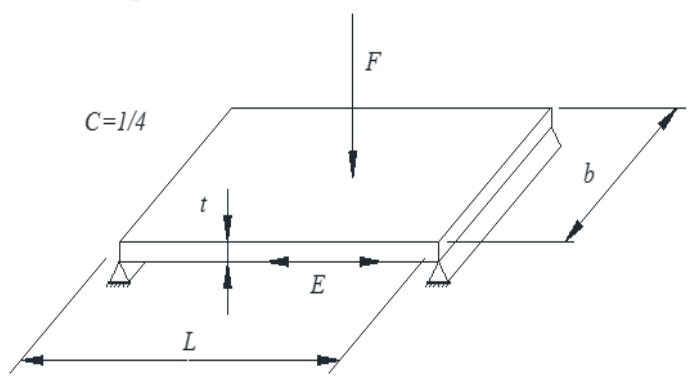

Fig.2 Deflection of a plate

The plate shown in Fig. 2 has a length of L, width of $b$ and depth of $t$, the original material is mild steel with the elastic modulus of $\mathrm{E}$, receive load $\mathrm{F}$ function on the plate.

As shown in Fig.1, the deflection at the center is

$$
w_{\max }=C \frac{F L^{3}}{E t^{3} b}
$$

Where, $\mathrm{C}$ is a constant, which takes into account the constraint condition, and $\mathrm{F}$ is the load. When the material is replaced, the stiffness should remain the same, so that the deflection should be kept constant.

Before the material is replaced, the bent of the center of the plate is:

$$
w_{\max }=C \frac{F L^{3}}{E_{S t} t_{S t}{ }^{3} b}
$$

After the mild steel sheet material is replaced by Aluminium alloy sheet of depth $t_{\mathrm{Al}}$ and elastic modulus $\mathrm{E}_{\mathrm{Al}}$, with other geometric conditions unchanged, we have:

$$
w_{\max }=C \frac{F L^{3}}{E_{A l} t_{A l}^{3} b}
$$

In order to maintain the deflection to be the same, we have:

$$
E_{A l} t_{A l}^{3}=E_{S t} t_{S t}^{3}
$$

Therefore, the depth of the aluminum plate should be: 


$$
t_{A l}=\sqrt[3]{\frac{E_{S t}}{E_{A l}}} t_{S t}
$$

It is often the ideal case in the above material analysis, material may fail under certain strain and most materials have strain rate effect of different magnitude, in order to ensure the validity of Eq.(8), one coefficient $\alpha$ is needed, then we obtain:

$$
t_{A l}=\alpha \sqrt[3]{\frac{E_{S t}}{E_{A l}}} t_{S t}
$$

The coefficient $\alpha$ is equal to approximately 1 , its value is correlative with the similarity of strain-stress, relations of mild steel and Aluminum alloy and the real parts' deformation. The coefficient $\alpha$ goes up with the increase of materials' failure strain, failure stress and strain rate sensitivity. The coefficient $\alpha$ may take the value of 1 where no high precision is required.

\subsection{Thickness selection of the same stability}

For the parameter selection of instability, the stiffness plays a major role in the original design as before.

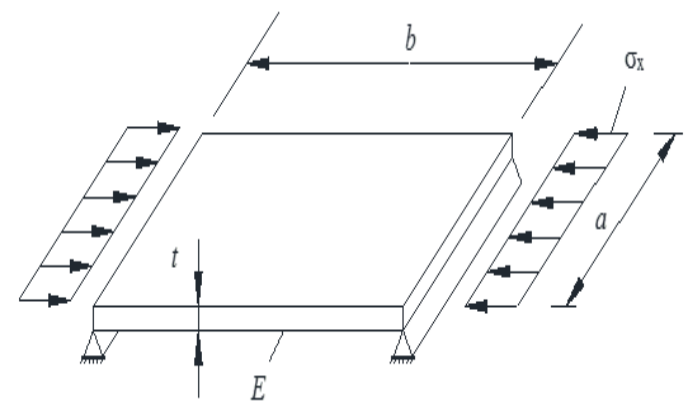

Fig.3 Plate heave

The plate shown in Fig. 3 has a length of $b$, width of a and depth of $t$, the original material is mild steel with the elastic modulus of $\mathrm{E}$, material strength is $\sigma_{\mathrm{x}}$.

In the case of a plate subjected to compressive stress, a bulge will occur in the center plane beyond a certain critical stress, which can be calculated as follows:

$$
\sigma_{X} \leq \sigma_{B}=k \frac{E t^{2}}{a^{2}}
$$

Here, $\mathrm{K}$ is the bump value associated with the actual situation, the geometric size and stress load is taking into account.

For rectangular fields, the value of the bump value is:

$$
k=\left(\frac{1}{\delta}+\delta\right)^{2}
$$

among the equation, $\delta=\mathrm{a} / \mathrm{b}$.

before the replacement material, the strength of the steel plate is:

$$
\sigma_{x} \leq \sigma_{B}=k \frac{E_{S t} t_{S t}{ }^{2}}{a^{2}}
$$


After the mild steel sheet material is replaced by Aluminium alloy sheet of depth $t_{\mathrm{Al}}$ and elastic modulus $\mathrm{E}_{\mathrm{Al}}$, with other geometric conditions unchanged, we have:

$$
\sigma_{X} \leq \sigma_{B}=k \frac{E_{A l}{ }_{A l}^{2}}{a^{2}}
$$

In order to maintain the uplift strength to be the same ,the depth of the aluminum plate should be:

$$
t_{A l}=\sqrt{\frac{E_{S t}}{E_{A l}}} t_{S t}
$$

In a similar way, in view of the inaccuracy of material model ,the above equation may be revised as:

$$
t_{A l}=\beta \sqrt{\frac{E_{S t}}{E_{A l}}} t_{S t}
$$

The coefficient $\beta$ is also equal to approximately 1 like the coefficient $\alpha$ in Eq. (9). The coefficient $\beta$ may take the value of 1 where no high precision is required.

Box body parts are mostly thin sheet stamping. The above analysis can be used to determine body part sheet depth after material replacement without a large error. If each part of Aluminum alloy steel has the same strength of the part of mild steel sheet, under the restriction of neighboring parts, the deformation mode and bulge of each part of Aluminum alloy sheet is nearly the same with that of mild steel. Therefore, for body parts which may have deformation under bending moment or plastic stretch under tension, the above analysis is applicable for lightweighting analysis.

Because $\alpha$ and $\beta$ are close to 1, the estimation of thickness of sheet as in Eq.(9) is less than that in Eq.(15). Therefore, the thickness of sheet determined by Eq. (15) could meet the demand of stiffness requirements for both cases.

\section{Electric car battery box structure and checking conditions}

The battery box of electric vehicle is composed of a series of parts which are made of sheet metal,in addition to the reserved openings, it forms a confined space,the battery box is connected with the electric vehicle chassis through bolts,the overall size of the battery box is designed to fit the installation space of the electric car, the size of the battery box is: $825 \mathrm{~mm} * 512 \mathrm{~mm} * 223 \mathrm{~mm}$, battery box structure as shown in figure 4 :

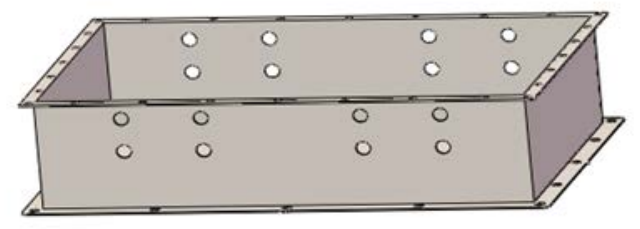

Figure.4 Battery box structure

The lithium battery module is placed inside the battery box,due to the instability of the 
road and the instability of driving, the dynamic force of the battery box is very complex.The force of the battery box can be broken down into three:(a)The impact force of the upper and lower directions on the bottom of the battery box ;(b)The impact force of the front and rear directions on the small side wall of the battery box;(c):The impact force of the left and right directions on the large side wall of the battery box.

\subsection{Analysis conditions of battery box bottom plate}

The total weight of the lithium battery module inside the battery box is $140 \mathrm{~kg}$, so the weight of the bottom of the battery box bears is $140 \mathrm{~kg}$,according to the technical requirements, the battery box must be able to withstand the gravity of 3g,therefore, the force acting on the bottom of the battery box is $4200 \mathrm{~N}$,the first condition that the battery box needs to be checked is if the strength of the bottom plate is enough under the pressure of internal weight $420 \mathrm{~kg}$ ?

\subsection{Analysis conditions of small side wall of battery box}

According to the technical requirements, the vehicle begin to brake when the electric vehicle speed is $100 \mathrm{~km} / \mathrm{h}$, in order to ensure that the braking distance is $40 \mathrm{~m}$, the mechanical strength should be ensured in such an acceleration shock,assumed initial velocity is $V_{0}=100 \mathrm{~km} / \mathrm{h}$, the final velocity is $V_{t}=0$, braking distance is $S=40 \mathrm{~m}$,by formula:

$$
-2 \mathrm{aS}=\mathrm{V}_{\mathrm{t}}^{2}-\mathrm{V}_{0}^{2}
$$

we can obtain: $\mathrm{a}=-\left(\mathrm{V}_{\mathrm{t}}^{2}-\mathrm{V}_{0}{ }^{2}\right) / 2 \mathrm{~s}=-(100 * 1000 / 3600)^{2} / 2 * 40 \approx-9.645 \mathrm{~m} / \mathrm{s}^{2}$

By Newtonian mechanics formula: $F_{1}=m a=140 * 9.645=1350.3 \mathrm{~N}$, therefore, in the braking process, the force on the small side wall of the battery box is $1350.3 \mathrm{~N}$. The safety factor should be considered in the design process, and the safety factor is set to 1.2 , therefore, $\mathrm{F}_{2}=1350.3 * 1.2=1620.36 \mathrm{~N}$, so the mechanical strength of the small side wall of the battery box is checked by the force of $1620.36 \mathrm{~N}$ on the small side wall.

\subsection{Analysis conditions of large side wall of battery box}

The impact force generated by the vehicle during acceleration or braking is mainly on the side wall of the front and rear directions,the impact force generated by the vehicle when turning or changing direction is mainly on the side wall of the left and right directions.

Due to the impact force generated when turning or changing is less than the impact acceleration or braking. Therefore, the impact force of the large side wall of the battery box is less than that of the small side wall of the battery box,For the safety, we also check the large side wall in accordance with the force of $1620.36 \mathrm{~N}$.

\section{Comparison and analysis of stress and displacement simulation of battery box}

The battery case is made of sheet metal and welded element,in order to reduce the amount of computation in simulation analysis, it is necessary to simplify the model.We remove the redundant parts of the battery box, simplify the structure of the mechanical strength of the bottom plate and the side wall, and get a suitable model for simulation analysis.

The box bears the weight of the battery and the weight of itself. The initial material is 
ordinary steel plate,the thickness of the box plate is $5 \mathrm{~mm}$, the others are $3 \mathrm{~mm}$ after material replacement, the thickness of the aluminum plate and other parts should be:

$$
\begin{gathered}
t_{A l_{2}}=\beta \sqrt{\frac{E_{S t}}{E_{A l}}} \cdot t_{S t 2}=8.66 \mathrm{~mm} \\
t_{A l 1}=\beta \sqrt{\frac{E_{S t}}{E_{A l}}} \cdot t_{S t 1}=5.12 \mathrm{~mm} \\
\mathrm{E}_{\mathrm{St}}=210000 \mathrm{MPa}, \mathrm{E}_{\mathrm{Al}}=72000 \mathrm{MPa} .
\end{gathered}
$$

The yield stress of steel is not less than $470 \mathrm{MPa}$ and the yield stress of aluminum alloy is $245 \mathrm{MPa}$.

Among the equation, $\beta$ is defined as $1(0 \leq \beta \leq 1)$.As the aluminum plate is a certain size, so the thickness of the aluminum plate rounded to $5 \mathrm{~mm}$ and $8 \mathrm{~mm}$.

\subsection{Simulation and analysis of the strength of the battery box bottom plate}

The upper and lower vibration of the car is big because of the pavement condition,the battery module has a greater impact on the bottom of the battery box,the lower case of the battery box is connected with the chassis through the shaft hole.Due to large amount of contact analysis, the model is simplified by equivalent load,we connect the battery box to the chassis as a stationary surface,then apply $4200 \mathrm{~N}$ to the underside of the battery case,load and boundary constraints are shown in figure 5:

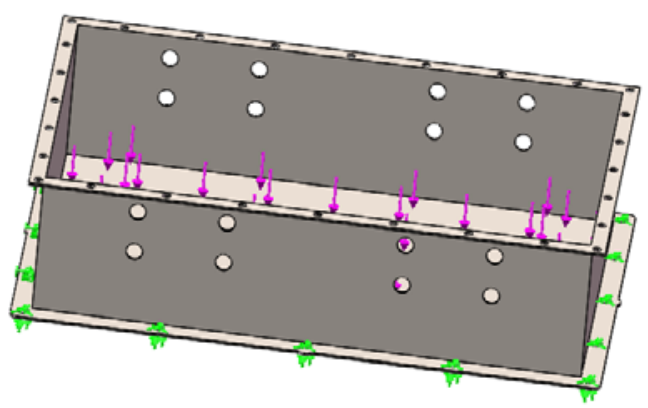

Fig.5 The load and boundary constraint diagram of the box bottom plate Through the simulation analysis, the stress and displacement of the steel plate battery box and Aluminum alloy battery box are shown in figure 6:

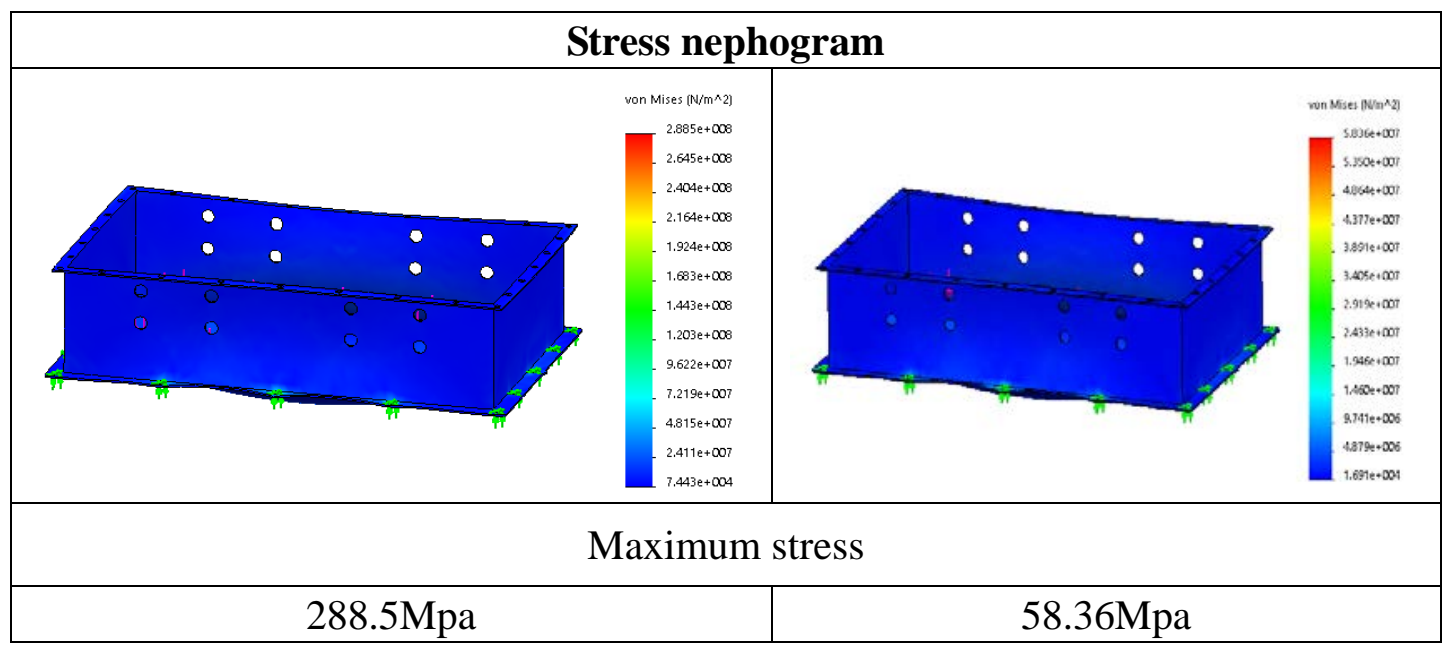




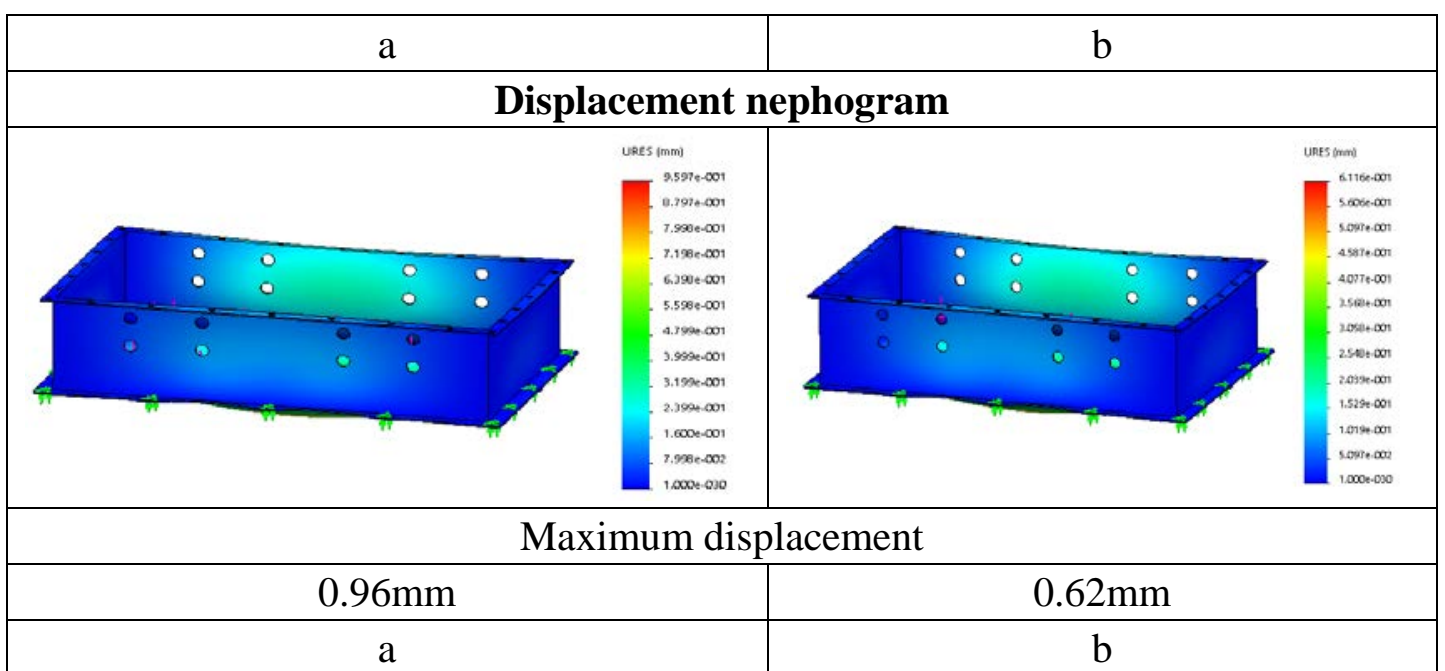

Fig.6 Stress nephogram and Displacement nephogram of bottom plate:(a) mild steel ;(b) Aluminum alloy

According to the stress distribution, the maximum stress is $288.5 \mathrm{Mpa}$ and $58.36 \mathrm{Mpa}$ respectively, which are distributed on the edge of the shaft hole, and the yield limit is not exceeded.As shown in the displacement distribution diagram, the maximum displacement is $0.96 \mathrm{~mm}$ and $0.62 \mathrm{~mm}$ respectively, and the deformation is small. The strength of the box can meet the requirements of the vehicle.

\subsection{Simulation and analysis of small side wall strength of battery box}

In the case of emergency braking,due to the inertia, the battery module inside the battery box will have a great impact on the battery box,the impact force is on the small side wall of the battery box.

According to the above calculation, the impact force of the battery module on the small side wall of the battery box is $1620.36 \mathrm{~N}$,due to the two small side walls are symmetrical, we only analyze one side.

We use the shaft hole as a stationary surface, and then apply the force of $1620.36 \mathrm{~N}$ to the interior side of the cell,load and boundary constraints are shown in figure 7 :

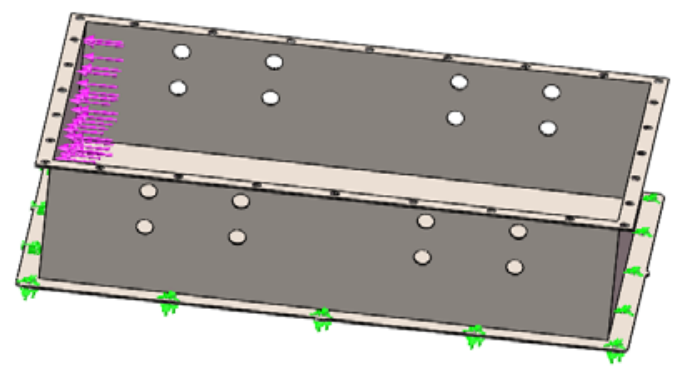

Fig. 7 Load and boundary constraint of the small side wall of the battery box Through the simulation analysis, the stress and displacement of the steel plate battery box and aluminum alloy battery box are shown in figure 8: 


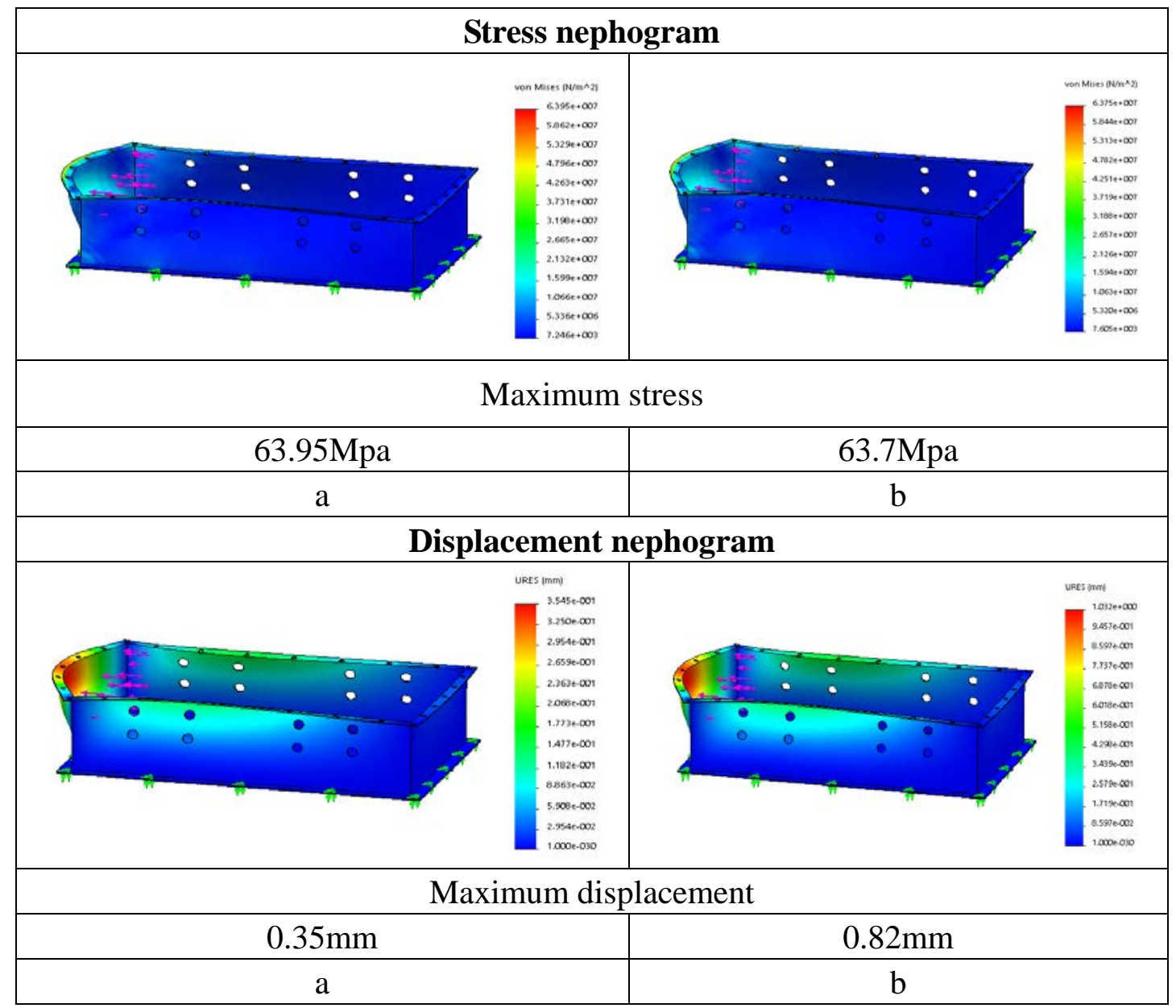

Fig.8 Stress nephogram and Displacement nephogram of small side wall:(a) mild steel ;(b) aluminum alloy

According to the stress distribution, the maximum stress is $63.95 \mathrm{Mpa}$ and $63.7 \mathrm{Mpa}$ respectively, which are distributed on the edge of the box, and the yield limit is not exceeded.According to the displacement distribution diagram, the maximum displacement is $0.35 \mathrm{~mm}$ and $0.82 \mathrm{~mm}$ respectively, and the deformation is small. The strength of the box can meet the requirements of the vehicle.

\subsection{Simulation and analysis of large side wall strength of battery box}

In the case of turning or changing the direction , the battery module inside the battery box will have a certain impact on the battery box,the impact force is on the large side wall of the battery box.

According to the above calculation, the impact of the battery module on the large side wall of the battery box is $1620.36 \mathrm{~N}$,since the two large side walls are symmetrical,we only analyze one side.

We use the shaft hole as a stationary surface, and then apply the force of $1620.36 \mathrm{~N}$ to the inside of the cell wall,load and boundary constraints are shown in figure 9: 


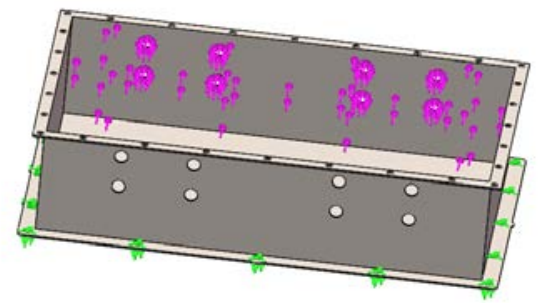

Fig. 9 Load and boundary constraint of the big side wall of the battery box Through the simulation analysis, the stress and displacement of the steel plate battery box and aluminum alloy battery box are shown in figure 10 :

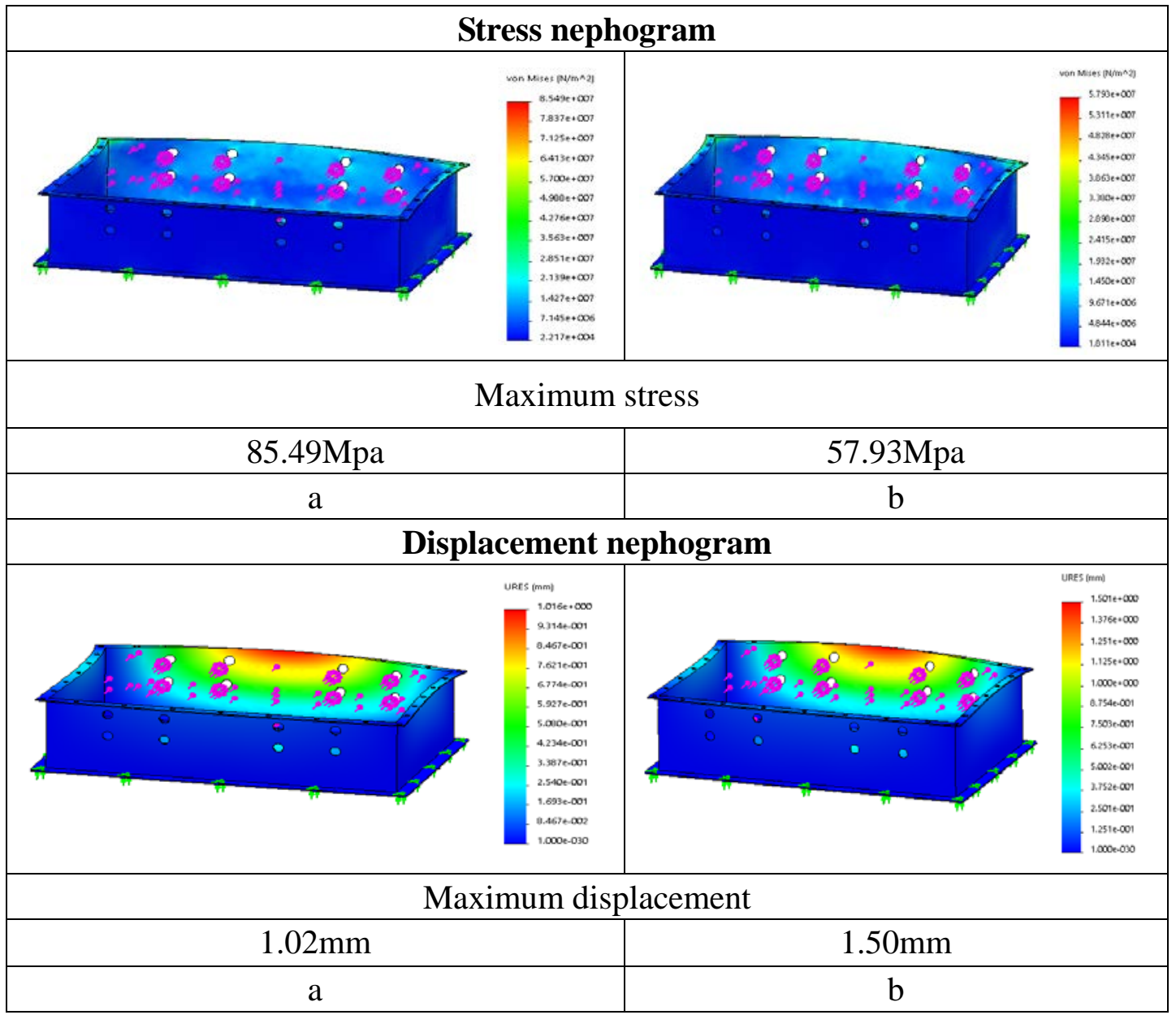

Fig.10 Stress nephogram and Displacement nephogram of large side wall:(a) mild steel ;(b) aluminum alloy

From the stress distribution diagram shows that the maximum stress is respectively 85.49Mpa and 57.93Mpa, are distributed in the box on the edge, do not exceed the yield limit of the material.From the displacement distribution diagram, the maximum displacement is respectively $1.02 \mathrm{~mm}$ and $1.50 \mathrm{~mm}$, the deformation is small,The strength of the box can meet the requirements of the vehicle.

Through the above simulation and analysis, we can draw: before and after the replacement of the material, the stress is not beyond the yield limit of the materials, the deformation is in a reasonable range, in line with the general requirements of electric vehicles. 
It can be seen that the difference between the displacement and the stress is relatively small when the material is replaced.

By use of Aluminum alloy sheet, the body weight is reduced. The weight reduction ratio of the parts after material replacement is:

$$
\begin{gathered}
\frac{G_{A l}}{G_{S t}} \approx \frac{\rho_{A l}}{\rho_{S t}} \cdot \frac{t_{A l}}{t_{S t}}=0.57 \\
\rho_{\mathrm{St}}=7.85 \mathrm{~g} / \mathrm{cm}^{3}, \rho_{\mathrm{Al}}=2.7 \mathrm{~g} / \mathrm{cm}^{3}, \mathrm{t}_{\mathrm{Al}}=5 \mathrm{~mm}, \mathrm{t}_{\mathrm{St}}=3 \mathrm{~mm} .
\end{gathered}
$$

The above calculation is only theoretical value, there is a little difference in the actual manufacturing process.The total weight of the parts for material replacement is $40 \mathrm{~kg}$,after the material replacement,the weight is $24 \mathrm{~kg}$,the weight reduction ratio is $40 \%$.Through the use of Aluminum alloy sheet, the body weight is reduced by $16 \mathrm{~kg}$ without impairment of the stiffness of the battery inner box. Therefore, the method brought forward in this paper for determining the depth of Aluminum alloy sheet for replacement of the mild steel sheet for automobile body lightweighting is applicable.

\section{Conclusions}

(1)After the actual operation and verification on the electric vehicle, no deformation, cracking and other anomalies, the actual effect is better, meet the requirements of vehicle operation. Verified by simulation analysis on the mechanical properties of the battery box structure, the design process has a reliable basis, avoid some uncertainty, which greatly improved the accuracy of the design, not only improve the efficiency of product design, shorten the design cycle, accelerate the product development process, but also improve the performance of the product, reduce the cost of product development.

(2)This paper brings forward a method which is using material index to select suitable materials in different mechanical situations is of great significance for practice.

(3)This paper brings forward the method of box body parts lightweighting using Aluminum alloy steel sheet of different depth to replace the original design of mild steel sheet based on strength analysis, it is proved that this method is feasible.

(4)The simulation analysis of battery box shows the effectiveness of the method. The results show that the box body weight is reduced while its stiffness is not reduced, the weight reduction ratio of the part after material replacement is $40 \%$, which means that the method of battery box lightweighting by Aluminum alloy sheet replacement brought forward in this paper is applicable.

\section{Acknowledgments}

This work was financially supported by International Science \& Technology Cooperation Program of China (Grant No.2014DFA80440).

Correspodning author:Qiu-Sheng Chen,born in 1987,is currently a PhD candidate at School of Mechanical Engineering,Hefei University of Technology,China.Research interests include mechanical transmissions,electrical vehicles,mechanical design etc. Email:874792703@qq.com. 
Han Zhao, is currently a professor at School of Mechanical Engineering, Hefei University of Technology,China. He received his PhD degree from Aalborg University, Danmark, in 1990. Research interests include mechanical transmissions, electrical vehicles, mechanical design etc.

Ling-Xue Kong,is currently a professor a professor at Institute for Frontier Materials, Deakin University,Australia.

Kang-Wei Chen,is currently a engineer at Battery System Department of New Energy Vehicle R\&D Institute, ANHUI JIANGHUAI AUTOMOBILE CO.,LTD.China.

\section{References}

[1]C Kimble,H Wang.China's new energy vehicles: value and innovation[J].Journal of Business Strategy,2013,34(2):13-20.

[2] WANG Wei Wen, BI Rong Hua, Technical basis of Electric vehicle[M]. Beijing, China Machine Press,2010:3 (In Chinese).

[3]DONG Xianglong, ZHANG Weiqiang,Intensity Analysis and Structural Improvement of Electric Vehicle Battery Box Based on Workbench[J].Jouranl of Mechanical Strength,2015,37(2):312-316.

[4]M Hartmann,M rosehitz,z Khalil. Enhanced Battery Pack for Electric Vehicle:Noise.reduction and Increased Stiffness[J].Materials Science Forum,2013, 818-822.

[5]Jambor Arno, Beyer Matthias. New cars—new materials. Material \& Design 1997,18:203-209.

[6]Cheon SS, Lee DG, Jeong KS. Composite side door impact beams for passenger cars. Composite Structure 1997,38:29-39. 